\title{
Efeito de dinamizações de Arnica montana L. no metabolismo de chambá (Justicia pectoralis Jacq.)
}

\author{
ANDRADE, F.M.C.*; CASALI, V.W.D.; CECON, P.R.C. \\ ${ }^{1}$ Universidade Federal de Viçosa-Departamento de Fitotecnia, Campus da UFV, s/n, CEP: 36570-000, Viçosa- \\ Brasil *fernandamcandrade@hotmail.com
}

\begin{abstract}
RESUMO: Este estudo objetivou avaliar a resposta do crescimento e do metabolismo secundário de Justicia pectoralis, expresso em produção de cumarina, a crescentes dinamizações de $A$. montana. O experimento foi conduzido na Universidade Federal de Viçosa. O delineamento estatístico foi inteiramente casualizado, com seis repetições e cinco tratamentos, totalizando 30 parcelas experimentais, sendo cada parcela constituída de uma planta por vaso. Os tratamentos foram as dinamizações $3 \mathrm{CH}, 30 \mathrm{CH}, 60 \mathrm{CH}, 100 \mathrm{CH}$ e $200 \mathrm{CH}$ do preparado homeopático A. montana. Os tratamentos foram aplicados às plantas via pulverização, em intervalos semanais, iniciando logo após o plantio. Após quatro meses do plantio as plantas foram colhidas. As características de crescimento avaliadas foram matérias fresca e seca de folhas e caules, matérias fresca e seca de inflorescências e matérias fresca e seca total. No estudo fitoquímico foi avaliada a produção da cumarina (1-2 benzopirona). Não houve resposta nas variáveis de crescimento aos tratamentos. As dinamizações de $A$. montana causaram alterações no metabolismo secundário das plantas. Os conteúdos de cumarina das plantas com $A$. montana $3 \mathrm{CH}$ e $30 \mathrm{CH}$ foram próximos e mais baixos, aumentando progressivamente a partir de $60 \mathrm{CH}$, chegando ao máximo em $100 \mathrm{CH}$, seguido de redução em $200 \mathrm{CH}$. A preparação homeopática $A$. montana causa alterações no metabolismo secundário de chambá, sendo as repostas dependentes da dinamização.
\end{abstract}

Palavras-chave: Justicia pectoralis, homeopatia, cumarina

ABSTRACT: Effect of dynamizations of Arnica montana in metabolism of chambá (Justicia pectoralis Jacq.). Were evaluated the responses to dynamizations of Arnica montana in the growth and in the secondary metabolism of Justicia pectoralis expressed as coumarin production. The studies were carried out at the Universidade Federal de Viçosa. The statistical design was completely randomized, with six replicates and five treatments, 30 experimental plots, one plant per pot. The treatments were dynamizations $3 \mathrm{CH}, 30 \mathrm{CH}, 60 \mathrm{CH}, 100 \mathrm{CH}$ and $200 \mathrm{CH}$ homeopathic preparation of $A$. montana. The application the treatments begun are planting seedlings, the aerial part being sprayed, at weekly intervals. After four months of planting, the plants were harvested. The evaluated growth characteristics were: fresh and dry matter of leaves and stems, fresh and dry matter of inflorecenses, and fresh and dry matter total. In the phytochemistry study, the production of the coumarin 1,2-benzopyrone was evaluated. Were no responses of growth characteristics. The dynamizations of $A$. montana caused changes in secondary metabolism of plants. The coumarin production with $A$. montana plants $3 \mathrm{CH}$ and $30 \mathrm{CH}$ were lower, increasing progressively from $60 \mathrm{CH}$, and coming to increased in $100 \mathrm{CH}$, followed by a large reduction in the $200 \mathrm{CH}$. The homeopathic preparation A. montana cause change in secondary metabolism of chambá, and the responses depend on dynamizations.

Key words: Justicia pectoralis, homeopathy, coumarin

\section{INTRODUÇÃO}

A maioria das substâncias bioativas encontradas nas plantas, normalmente são provenientes do metabolismo secundário. Aquantidade e a qualidade dos metabólitos secundários estão relacionadas com componentes genéticos e ambientais (Andrade \& Casali, 1999).

O cultivo das espécies medicinais, além de minimizar o extrativismo e até mesmo a extinção de

Recebido para publicação: setembro de 2011

Aceito para publicação: março de 2012

Rev. Bras. PI. Med., Botucatu, v.14, n.esp., p.159-162, 2012. 
espécies, visa à maior previsibilidade da qualidade fitoquímica das plantas, uma vez que diferentes práticas de manejo interferem diretamente nos processos de síntese dos metabólitos secundários (Silva et al., 2010).

No cultivo de espécies medicinais é recomendado o modelo orgânico/ecológico de produção, visando à preservação da qualidade biológica das plantas. A qualidade biológica refere-se à composição interna equilibrada, mas também a ausência de resíduos químicos provenientes de adubações e agrotóxicos (Casali et al., 2010).

A homeopatia é insumo recomendado à produção orgânica no Brasil (Brasil, 1999). As preparações homeopáticas por serem dinamizadas não deixam resíduos nas plantas e causam alterações mais evidentes no metabolismo secundário das espécies medicinais em relação ao metabolismo primário (Casali et al., 2000).

A espécie medicinal J. pectoralis Jacq. ocorre naturalmente nas Américas do Sul, do Norte, Central e Tropical, no Oeste da África e no Oeste da Índia (Barros, 1992). No Brasil, ocorre espontaneamente no estado do Amazonas, território de Roraima, em margem de rios e em florestas secundárias (Lino et al., 1997).

Justicia pectoralis, conhecida popularmente como chambá, pertence à família Acanthaceae. As cumarinas são metabólitos secundários de defesa elaborados pela planta "chambá". As cumarinas exercem ação terapêutica em sistemas biológicos (Barros, 1992).

Segundo Andrade et al. (2001), a produção de cumarina em chambá é alterada pela aplicação da homeopatia A. montana $3 \mathrm{CH}$.

Entretanto, a ação da homeopatia em organismos vivos depende não apenas da escolha da preparação homeopática, mas também da dinamização (Casali et al., 2006). De acordo com Bonfim et al. (2008), a homeopatia A. montana causa alterações no enraizamento das espécies medicinais Rosmarinus officinalis L. e Lippia alba [(Mill.) N.E. Brown], sendo as respostas dependentes da dinamização.

Este trabalho teve por objetivo estudar a ação de dinamizações da homeopatia Arnica montana no metabolismo de chambá.

\section{MATERIAL E MÉTODO}

As mudas foram propagadas por divisão de touceiras de plantas matrizes pertencentes ao Grupo Entre Folhas, localizado em Viçosa-MG, sendo diretamente plantadas em vasos com capacidade de três litros, contendo como substrato terra de mata, sem adubação. A exsicata encontra-se no Herbário VIC do Departamento de Biologia Vegetal da
Universidade Federal de Viçosa (UFV), identificada pelo número VIC 23581.

O experimento foi conduzido em Viçosa MG nas dependências do telado do Departamento de Fitotecnia, na Universidade Federal de Viçosa, durante o período de junho a outubro de mil novecentos e nove e nove. Aárea recebeu $100 \%$ de luminosidade, por ser mais adequada à espécie, visando maior produção de cumarinas (Barros, 1997).

O delineamento experimental foi inteiramente casualisado, com seis repetições e cinco tratamentos, totalizando 30 parcelas experimentais, sendo cada parcela constituída de uma planta/vaso. Os vasos de cada tratamento foram distanciados de $3 \mathrm{~m}$, isolados com filme de polietileno transparente e distribuídos aleatoriamente, sendo feito rodízio quinzenalmente.

Os tratamentos foram as dinamizações 3 , $30,60,100$ e 200, na escala centesimal hahnemaniana $(\mathrm{CH})$, da homeopatia $A$. montana. Na implementação dos tratamentos, sob procedimento Duplo Cego, que iniciou após o plantio, foi preparada a solução contendo 10 gotas da homeopatia/litro de água desmineralizada. O Duplo Cego é adotado nos experimentos de Homeopatia e prevê a codificação dos tratamentos visando ocultá-los do pesquisador. Os códigos são revelados após a análise dos dados, visando minimizar interferências. A solução homeopática foi pulverizada, aproximadamente 2,65 $\mathrm{mL}$ planta-1 $^{-1}$, à parte aérea das plantas, em intervalos semanais, nas primeiras horas do dia, durante todo período experimental. As homeopatias foram adquiridas em Laboratório Comercial.

Após quatro meses do plantio as plantas foram colhidas com auxílio de tesoura de poda. Imediatamente após a colheita, foi determinado em balança semi - analítica o peso da matéria fresca das folhas e caules, das inflorescências, e o peso da matéria fresca total. Após a determinação do peso da matéria fresca, as plantas foram embaladas em sacos de papel "Kraft", devidamente identificados e, conduzidas à câmara de secagem. As plantas foram secas em sala com desumidificador (capacidade 18 $\mathrm{L}$ dia-1 $280 \mathrm{~m}^{-3}$, circulação $60 \mathrm{~m}^{2} / \mathrm{h}, 390$ watts) com temperatura entre 15,4 e $20,9^{\circ} \mathrm{C}$, até atingirem peso constante, quando foram pesadas em balança semi - analítica, obtendo-se o peso da matéria seca das folhas e caules, das inflorescências e o peso da matéria seca total.

Após a secagem, as amostras foram acondicionadas em saco de polipropileno, sem ar e vedados com fita adesiva, devidamente identificadas, e acondicionadas em saco preto, em geladeira à $10^{\circ} \mathrm{C}$, até a realização das análises químicas.

A extração e quantificação das cumarinas foram realizadas no Laboratório de Análise e Síntese de Agroquímicos do Departamento de Química da Universidade Federal de Viçosa. 
Foram pesadas amostras de $200 \mathrm{mg}$ da parte aérea (folhas e caules) seca da planta que foram reduzidas a pó em moinho Marconi, modelo TE 048, as quais foram submetidas a três extrações a quente $\left(65^{\circ} \mathrm{C}\right)$ em $7 \mathrm{~mL}$ de metanol, e após filtragem foram concentradas em evaporador rotativo. O resíduo obtido foi dissolvido em $10 \mathrm{~mL}$ de água destilada e $10 \mathrm{~mL}$ de diclorometano. A fase orgânica foi separada por meio de funil de separação e em seguida seca sobre sulfato de magnésio anidro. Após filtração a vácuo, as amostras foram armazenadas em vidros vedados com parafilme e guardadas em temperatura de $\left(-10^{\circ} \mathrm{C}\right)$ até o momento da quantificação das cumarinas.

As amostras obtidas na extração e armazenadas à baixa temperatura $\left(-10^{\circ} \mathrm{C}\right)$ tiveram o volume completado à $5 \mathrm{~mL}$ com diclorometano. Deste volume foram retirados $200 \mu \mathrm{L}$ e adicionados $2 \mathrm{~mL}$ de diclorometano, obtendo a amostra que foi utilizada na quantificação da cumarina.

A quantificação da cumarina (1,2benzopirona) foi realizada em Cromatógrafo Líquido de Alta Eficiência (HPLC). O volume injetado de amostra foi de $20 \mu \mathrm{L}$, utilizando como fase móvel acetato de etila:hexano (3:1), com fluxo de 1,2 mL $\mathrm{min}^{-1}$. Com base em procedimentos de ajuste, o tempo de retenção da cumarina foi aproximadamente 5,3 minutos e a concentração nas amostras foi calculada mediante a curva padrão obtida pela injeção no HPLC de soluções padrão de 1,2-benzopirona (MERCK), em concentrações conhecidas de 0, 2, 4, 6, 8 e 10 ppm.

Os dados foram interpretados por meio de análise de variância e regressão. Os modelos foram escolhidos baseados na significância dos coeficientes de regressão utilizando o teste de " $t$ " ao nível de 5\% de probabilidade.

\section{RESULTADO E DISCUSSÃO}

As crescentes dinamizações de A. montana não causaram efeitos significativos nas variáveis que quantificaram o crescimento das plantas (Tabela 1).

Segundo Armond et al. (2005), em picão, as dinamizações de China, causaram alterações no conteúdo de óleo essencial das plantas, sem no entanto, causar alterações adaptativas ao ambiente na morfologia externa.

O metabolismo secundário do chambá

TABELA 1. Resumo da análise de variância das variáveis: matéria fresca de folhas e caules (MFFC), matéria fresca das inflorescências (MFI), matéria fresca total (MFT), matéria seca de folhas e caules (MSFC), matéria seca das inflorescências (MSI) e matéria seca total (MST) de Justicia pectoralis, colhida no ensaio conduzido no período de junho a outubro de 1999, realizado em Viçosa-MG.

\begin{tabular}{|c|c|c|c|c|c|c|c|}
\hline \multirow{2}{*}{ F.V. } & \multirow{2}{*}{ GL. } & \multicolumn{6}{|c|}{ Quadrado Médio } \\
\hline & & MFFC & MFI & MFT & MSFC & MSI & MST \\
\hline Tratamento & 4 & $3,0986^{\mathrm{ns}}$ & $6,2669^{\text {ns }}$ & $11,0710^{\text {ns }}$ & $8,3938^{\text {ns }}$ & $21,4568^{\text {ns }}$ & $4,9616^{\text {ns }}$ \\
\hline Resíduo & 25 & 12,3155 & 5,7915 & 6,8256 & 6,1153 & 10,2152 & 16,3981 \\
\hline C.V. (\%) & & 39,53 & 50,44 & 19,14 & 7,96 & 9,52 & 6,26 \\
\hline
\end{tabular}

${ }^{\text {ns }} \mathrm{F}$ não significativo a $5 \%$ de probabilidade.

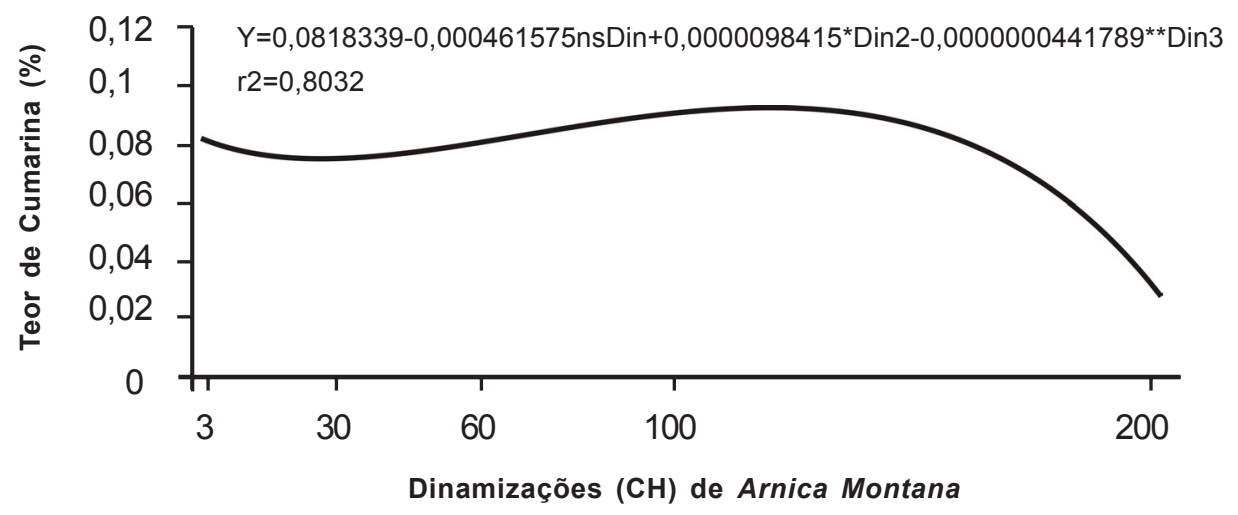

FIGURA 1. Teor de cumarina em Justicia pectoralis, em função das dinamizações da homeopatia Arnica 
respondeu às dinamizações de $A$. montana (Figura 1). Os conteúdos de cumarina das plantas $\operatorname{com} A$. montana $3 \mathrm{CH}$ e $30 \mathrm{CH}$ foram próximos e mais baixos, aumentando progressivamente a partir de $60 \mathrm{CH}$, chegando ao máximo em $100 \mathrm{CH}$, seguido de grande redução em $200 \mathrm{CH}$.

O modelo de equação de regressão, que mais correspondeu à produção de cumarina foi o modelo cúbico. $\mathrm{O}$ efeito ora estimulante ora supressor com o aumento da dinamização (respostas em forma de ondas) é modelo de resposta comum na experimentação homeopática em plantas (Silva, 2005; Casali et al., 2006). Segundo Andrade (2000), em plantas, a resposta ao aumento das dinamizações não implica necessariamente em aumento da reação, ou seja, o aumento das dinamizações não provoca respostas fisiológicas progressivas.

As preparações homeopáticas a partir da dinamização $12 \mathrm{CH}$ são desprovidas de moléculas. Cabe ressaltar o efeito das diluições situadas além do número de Avogadro no metabolismo secundário de J. pectoralis, resultado coerente com a hipótese de ação físico dinâmica, e não química, das ultradiluições (Casali et al., 2006).

Portanto, as preparações homeopáticas de A. montana atuaram como fator externo sobre o metabolismo secundário das plantas, causando alterações no conteúdo de cumarina, sendo a resposta dependente da dinamização. Os metabólitos secundários são bons sinalizadores dos efeitos das preparações homeopáticas em plantas.

\section{AGRADECIMENTO}

Ao CNPq, a Universidade Federal de Viçosa, aos Departamentos de Fitotecnia, Química e Informática.

\section{REFERÊNCIA}

ANDRADE, F.M.C.; CASALI, V.W.D. Plantas medicinais e aromáticas: relação com o ambiente, colheita e metabolismo secundário. Viçosa: UFV, 1999. 139p. ANDRADE, F.M.C. Homeopatia no crescimento e na produção de cumarina em chambá Justicia pectoralis Jacq. 2000. 214p. Dissertação (Mestrado- Área de Concentração em Fitotecnia) - Departamento de Fitotecnia, Universidade Federal de Viçosa, Viçosa.

ANDRADE, F.M.C. et al. Efeito de homeopatias no crescimento e na produção de cumarina em chambá (Justicia pectoralis Jacq.). Revista Brasileira de Plantas Medicinais, v.4, p.19-27, 2001.

BARROS, R.F.M. Efeito da radiação solar sobre o crescimento e a produção de cumarinas em Justicia pectoralis var. stenophylla Leonard. 1992. 160p. Dissertação (Mestrado-Área de Concentração em Biologia Vegetal) - Departamento de Bilogia Vegetal, Universidade Federal de Pernambuco, Recife.

BARROS, R.F.M.; ANDRADE, L.H.C.; SILVA, N.H. Concentração de cumarinas em folhas de Justicia pectoralis var. stenophylla Leonard com diferentes colorações. Oyton, v.60, p.141-5, 1997.

BONFIM, F.P.G. et al. Use of homeopathic Arnica montana for issuance of roots of Rosmarinus officinalis L. and Lippia alba (Mill.) N.E.Br. International Journal High Dilution Research, v.7, p.113-7, 2008.

BRASIL. Instrução normativa no 07 , de 17 de maio de 1999. Dispõe sobre normas para a produção de produtos orgânicos vegetais e animais. Diário Oficial [da República Federativa do Brasil], Brasília, v.99, n.94, p.1114, 19 maio 1999. Seção 1.

CASALI, V.W.D. et a. Homeopatia bases e princípios. Viçosa: UFV, 2006. 150p.

CASALI, V.W.D. et al. Benefícios da homeopatia no cultivo de plantas medicinais. Informe Agropecuário, v.31, p.7984, 2010.

LINO, C.S. et al. Analgesic and antiinflammatory activities of Justicia pectoralis Jacq. and its main constituents: coumarin and umbelliferone. Phytotherapy Research, v.11, p.211-5, 1997.

SILVA, M.B. et al. Uso de princípios bioativos de plantas no controle de fitopatógenos e pragas. Informe Agropecuário, v.31, p.70-7, 2010.

SILVA, M.R.B. Assimilação de $\mathrm{CO}_{2}$ em plantas de Sphagneticola trilobata (L.) Pruski tratadas com preparados homeopáticos. 2005. 54p. Dissertação (Mestrado - Área de Concentração em Fitotecnia) Departamento de Fitotecnia, Universidade Federal de Viçosa, Viçosa. 